\title{
VALVE DISEASE
}

\section{Endocarditis: basics}

S J Eykyn

Department of Microbiology, St Thomas' Hospital, London, UK

$\mathrm{M}$

iological expertise is essential in the diagnosis, management, and prevention of infective endocarditis (IE). Unfortunately old habits die hard and there are still doctors who persist in referring to this infection as "SBE" (subacute bacterial endocarditis) whether the patient has been ill for days, weeks or months, and think that it is generally caused by a microbe they know as "Strep viridans" and can often be blamed on dentists. IE cannot be considered as a homogeneous infection. It may arise in the community or, increasingly, in hospital or as a result of procedures undertaken in hospital; it may affect native valves (previously normal or abnormal) or prosthetic valves and may occur in intravenous drug users (IVDU) as well as those who do not use drugs. Although overall most cases of IE are caused by staphylococci, streptococci, and enterococci, the incidence of each group of organisms differs in the various types of IE. A wide variety of organisms account for the infections not caused by these three genera, and virtually every organism known to microbiologists has been reported to cause IE, albeit very rarely. At St Thomas' Hospital, in some 650 cases of IE seen over 30 years we have encountered infections caused by Erysipelothrix rhusiopathiae, Listeria monocytogenes, Campylobacter fetus, Lactobacillus rhamnosus, and Histoplasma capsulatum!

\section{Native valve endocarditis}

Native valve endocarditis (NVE) is the most common type of IE. The affected valve may be previously normal or abnormal, and the infection is usually acquired in the community but increasingly is also acquired in hospital.

\section{Community acquired NVE}

Community acquired NVE is now as likely to be caused by staphylococci, usually Staphylococcus aureus but sometimes by coagulase negative staphylococci, as it is to be caused by oral ("viridans") streptococci (fig 1), most commonly those of the sanguis and oralis groups. Enterococci (until quite recently known as streptococci) are less common but their incidence is increasing; most are Enterococcus faecalis. A trivial predisposing skin lesion is occasionally detected in staphylococcal IE and there may be poor dentition in oral streptococcal IE, but seldom relevant preceding dentistry. Staphylococci, even some coagulase negative strains such Staphylococcus lugdunensis, are virulent bacteria and are as likely to attack a previously normal valve as an abnormal one, whereas oral streptococci and enterococci are much less virulent and seem only to infect previously abnormal valves.

\section{Hospital acquired or hospital associated} NVE

These infections are almost always caused by staphylococci, usually $S$ aureus but occasionally coagulase-negative staphylococci, most often Staphylococcus epidermidis. The inexorable rise in methicillin resistant $S$ aureus (MRSA) in UK hospitals over the last decade has been paralleled by an increase in hospital acquired MRSA endocarditis. Most cases of hospital acquired NVE result from intravascular access site infections, even those used for peripheral venous access. Intravascular access site infection is especially common in patients on haemodialysis. Pacemaker associated NVE is also increasingly encountered.

\section{Prosthetic valve endocarditis}

Any prosthetic valve-whether mechanical or bioprosthetic - can become infected and the risk of infection is life long, with some infections occurring over 20 years after valve replacement. Although for many years it has been conventional to classify prosthetic valve endocarditis (PVE) as early (occurring within 60 days of valve surgery and acquired in the theatre or soon thereafter perhaps on the intensive care unit) or late (occurring more than 60 days after valve surgery and presumed to have been acquired in the community), these definitions are unsatisfactory. Infections acquired in the theatre, and particularly those caused by relatively avirulent bacteria such as $S$ epidermidis and corynebacteria, may present many months or even a year or more after surgery, and as with native valves, prosthetic valves can become infected from intravascular access sites at any time after implantation. Thus it may be preferable to classify PVE, as with NVE, as hospital acquired or community acquired. PVE acquired in hospital is predominantly caused by staphylococci, often coagulase negative strains, whereas infections acquired in the community have a similar range of pathogens as community acquired NVE but a higher incidence of unusual organisms.

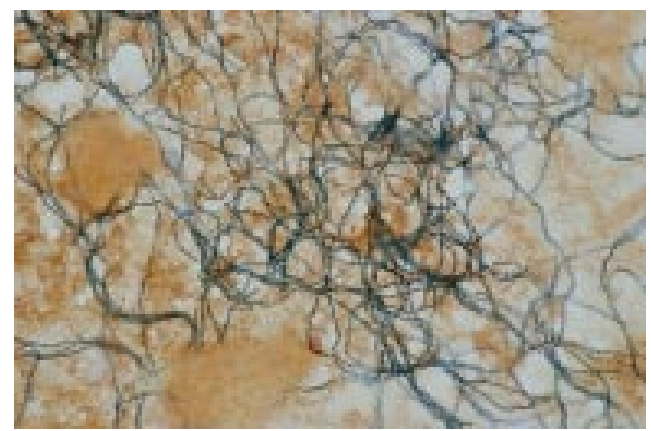

Figure 1. Gram stained smear from blood culture bottle showing viridans streptococci from patient with native valve endocarditis. 
Infective endocarditis in intravenous drug users

This infection, which usually involves the tricuspid valve, is frequently misdiagnosed as pneumonia by those unfamiliar with the respiratory presentation of right sided IE. Most cases are caused by $S$ aureus (fig 2).

\section{Microbiological diagnosis and monitoring of IE}

The mainstay of the microbiological diagnosis of IE is the blood culture. Not surprisingly, persistent positive blood cultures constitute a major diagnostic criterion on the Duke classification system ${ }^{1}$ now universally recognised as a means of confirming a definite case of IE. For some organisms their very presence in both bottles of a single blood culture more or less equates with a diagnosis of IE, and examples of this include many oral streptococci, Streptococcus bovis, and community acquired enterococci, but for others the demonstration of a persistent bacteraemia is required for diagnostic reassurance. Hence the convention is for several sets of blood cultures to be taken if IE is suspected. However, in practice in many cases of IE caused by virulent bacteria, especially $S$ aureus, the patient is recognised to be septic and unwell (fig 3) when first seen though IE is rarely suspected, and only a single set of blood cultures is done before broad spectrum antibiotics are started. There is no point in waiting for a spike of temperature before taking blood for culture, or taking cultures at specific time intervals or from different sites. Previous antibiotics will rarely prevent recovery of $S$ aureus from the blood but are very likely to prevent recovery of oral streptococci. It is seldom worth waiting 24-48 hours after stopping antibiotics before doing blood cultures. In most cases of IE the causative pathogen will be recovered from blood cultures within about 48 hours and most if not all bottles are positive. Recovery of an organism may take longer if antibiotics have been given or if fastidious organisms such as those known as the HACEK group (this includes Haemophilus species (though not Haemophilus influenzae), Actinobacillus actinomycetemcomitans, Cardiobacterium hominis, Eikenella corrodens, and Kingella kingae) are involved.

Although not strictly microbiological and not specific to IE, it is worth mentioning here that C-reactive protein (CRP) and the erythrocyte sedimentation rate (ESR) will usually be raised in IE and these have been proposed as additional minor criteria to the Duke classification of IE. ${ }^{2}$ The peripheral white blood cell count is usually very high in infections caused by virulent organisms but often normal in those caused by oral streptococci; the haemoglobin is usually low in the latter though not in the former. There may be microscopic haematuria and when this is not associated with a urethral catheter, urinary infection, end stage

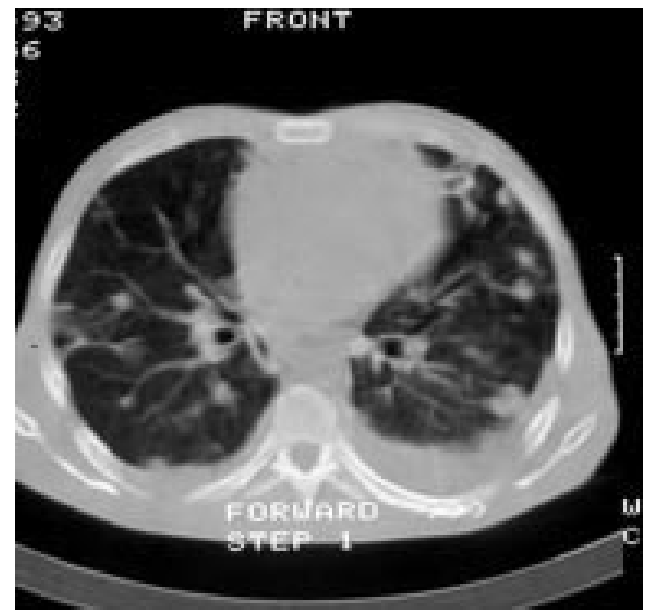

Figure 2. Computed tomographic chest scan from intravenous drug user with Staphylococcus aureus endocarditis on the tricuspid valve. Multiple cavitating lesions are evident.

renal disease or menstruation has been proposed as an additional minor criterion to the Duke classification. $^{2}$

It is only worth taking blood cultures during treatment of IE if the patient is febrile or unwell; "check" cultures to see if the blood has been sterilised when the patient is doing well are pointless. Serial CRP estimations can give laboratory reassurance that the infection is under control but the ESR often falls so slowly that it is much less useful in monitoring response to treatment than the CRP. Titrations of the serum bactericidal activity against the infecting organism ("back titrations") are of very limited value in monitoring antibiotic treatment, and at worst can produce false reassurance of bacteriological efficacy which may be accompanied by clinical deterioration. They should be abandoned.

\section{Blood culture negative IE}

In a variable percentage of cases where there is convincing clinical and echocardiographic evidence of IE the blood cultures are negative. In such cases it is essential to send blood for antibodies to bacteria that cannot be cultured by routine blood culture methods, specifically Coxiella burnettii (Q fever), Chlamydia species, and Bartonella species. A detailed history may reveal possible clues to these infections and it is worth noting that Bartonella species cross react with Chlamydia species. Also important in blood culture negative cases is a careful history of previous antibiotic administration which may necessitate a call to the general practitioner, because if antibiotics have been given and blood culturees are negative the pathogen is likely to be an oral streptococcus. Occasionally in blood culture negative IE the pathogen can be isolated from an excised valve or embolus, or sometimes detected on microscopy of such material even if this is sterile on culture. Blood cultures are seldom negative in intravenous drug users with IE, but if they are 
the pathogen can usually be isolated from respiratory specimens. When all these considerations have been taken into account, there really is no clue to the pathogen in less than $5 \%$ of patients with blood culture negative IE.

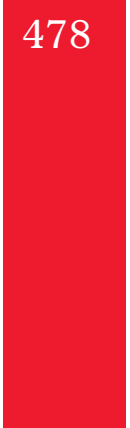

When should antibiotic treatment be started in suspected IE, and what with?

When a patient with suspected IE has been unwell for many weeks or months it is reasonable to wait $48-72$ hours to see if the blood cultures are positive before starting antibiotics, and then appropriate treatment can be given from the outset for the organism isolated. If the blood cultures are negative then the investigations outlined above should be done without delay and their results will determine treatment. The UK guidelines devised by the working party on endocarditis of the British Society for Antimicrobial Chemotherapy (BSAC) should be used. ${ }^{3}$ In those patients who are very unwell with suspected IE then obviously treatment should not be delayed after blood cultures have been taken and a combination such as vancomycin and gentamicin given. In reality endocarditis caused by virulent organisms is seldom diagnosed on admission and the initial broad spectrum antibiotic can be modified in light of culture results. Much mystique is attached to the value of in vitro tests on the pathogen by many microbiologists and the MIC (minimum inhibitory concentration) has long been the sacred cow of laboratory management. Not only are routine (disc) sensitivity tests quite adequate in almost all cases but treatment is generally well under way by the time in vitro tests are available. At least the MBC (minimum bactericidal concentration) has now been deemed of no value and with luck abandoned even by enthusiasts with time to spare.

\section{Length of treatment}

Many doctors are still convinced of the need for six weeks antibiotic treatment in all cases of IE, yet 20 years ago successful short course (two weeks) therapy for sensitive streptococcal infection was reported. ${ }^{4}$ Bacteriological failure - that is, recurrence of the infection - in IE generally means that surgery, not more antibiotics, is needed. There are distinct benefits for the patient from shorter courses of antibiotics as toxicity and intravenous access infection are less likely and usually the hospital admission shorter. There are no trials of short course treatment for IE caused by organisms other than oral streptococci; here the treatment is often complicated by the need for surgery and this may occur soon after the start of antibiotics. It is remarkable that surgery, even in the face of persistent infection and sometimes positive blood cultures, can be so dramatically curative. The length of treatment in most cases
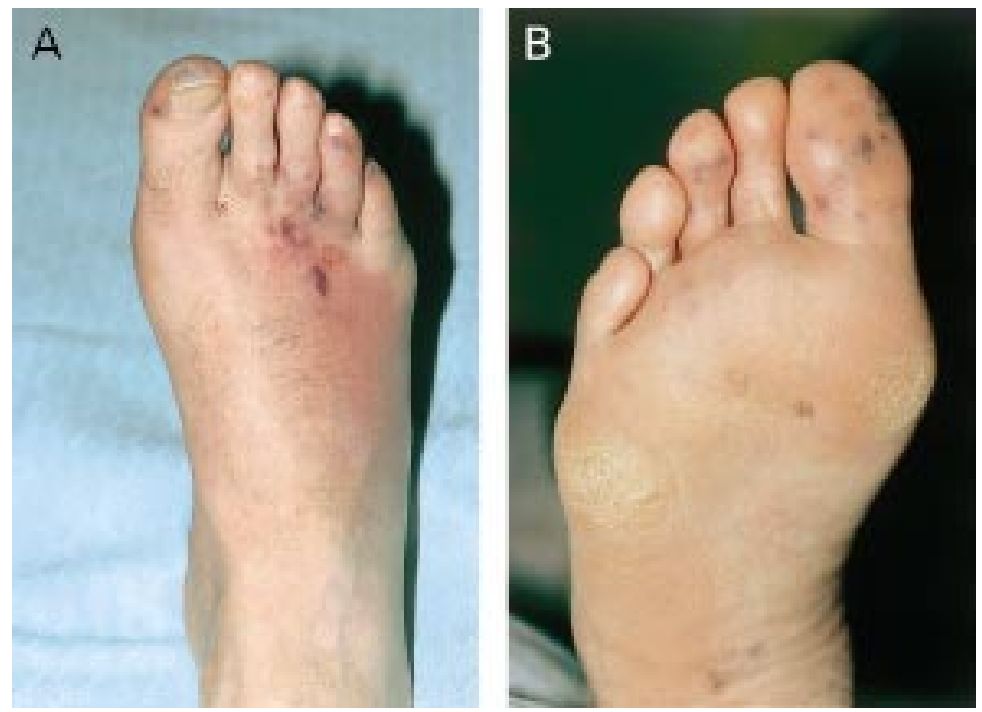

Figure 3. Embolic lesions on the feet of a patient with Staphylococcus aureus endocarditis.

who have emergency surgery can be determined by microscopy and culture of the excised valve. If the pathogen is isolated from the valve (and this may happen with $S$ aureus IE even after more than a week of appropriate antibiotics) then two weeks of intravenous antibiotics should be given after surgery. If the valve culture is sterile but organisms are detected on a Gram stained smear then the bacteria are dead; the intravenous antibiotics should be continued until the valve culture has been incubated for five days then they can be stopped. If no bacteria are detected on the Gram stained smear and the culture is sterile (cultures are likely to be sterile if no bacteria are seen on the smear) then there is no need for further antibiotic treatment.

\section{Choice of antibiotic for specific pathogens and the need for two agents}

The BSAC guidelines have already been mentioned. However they are only guidelines and in the individual case other regimens may be appropriate. It is quite possible, for example, to treat sensitive oral streptococcal IE in patients who are haemodynamically stable entirely by oral amoxicillin, provided the drug is taken reliably and absorbed. Likewise outpatient regimens have been used, though not I suspect in the UK. The long acting cephalosporin ceftriaxone has been successfully used in this way, usually given with an aminoglycoside. As has previously been mentioned, recurrence in IE is very seldom attributable to inadequate antibiotic treatment.

Most oral streptococci can be treated with a single agent (penicillin or amoxicillin), but there are no trials to show that a two week regimen with a single agent is appropriate and so it should be given for four weeks. The combination of an aminoglycoside (gentamicin but previously streptomycin) was originally devised to 
achieve bactericidal synergy against enterococci, as these bacteria cannot be eradicated with a penicillin alone. The combination is also recommended for oral streptococci on the basis of the reports of its successful use in two week regimens, even though for most oral streptococci there is no in vitro evidence of synergy. It is possible that two weeks of penicillin alone might be as effective, but this is unproven and thus cannot be recommended. There are unfortunately increasing numbers of enterococci with high level resistance to gentamicin and often also to streptomycin; therefore, the addition of the aminoglycoside to penicillin or amoxicillin will not result in synergy. For these strains there is currently no bactericidal regimen and prolonged courses of high dose amoxicillin are used, though in many cases surgery will be curative. Vancomycin resistant enterococci also occasionally cause IE; if these also show high level gentamicin resistance they pose a formidable therapeutic problem in patients allergic to penicillin.

It is conventional to treat staphylococcal IE with two antibiotics though there is very little evidence that this is beneficial. Trials of different treatment regimens in staphylococcal IE are almost impossible to assess because so many patients come to emergency surgery and it is this, not the antibiotics, that cures them. The addition of gentamicin to the $\beta$ lactam for two weeks in staphylococcal IE reduced the duration of fever and bacteraemia by about 24 hours in both IVDU and non-IVDU, but had no effect on morbidity or mortality. ${ }^{5}$ Fusidic acid is sometimes given in combination with flucloxacillin for staphylococcal IE, but benefit over the single agent is anecdotal. Rifampicin is often recommended in combination with vancomycin for MRSA or coagulase negative staphylococcal IE, especially PVE, but again convincing evidence of efficacy is lacking.

Persistence or recurrence of fever during appropriate antibiotic treatment of IE

While many patients with IE, particularly that caused by oral streptococci, respond within about 48 hours to antibiotic treatment with rapid resolution of fever, amelioration of many systemic symptoms and a decrease in the markers of bacterial infection, specifically CRP, some do not and this is cause for concern. ${ }^{6}$ Unless the symptoms can be attributed to drug hypersensitivity the antibiotic regimen should not be changed. The last thing such patients need, but often get, is an increased dose of antibiotic, an additional agent or a different regimen. Further blood cultures should be done and if the original pathogen is isolated then this in itself means that surgery is indicated. In practice only virulent bacteria such as $S$ aureus are likely to be recovered from blood cultures during antibiotic treatmentoral streptococci never will. Infected intravenous access sites are rarely responsible for persistent fever but should be carefully inspected nonetheless. By far the most common cause of fever is an abscess of the valve ring and surrounding structures or widespread tissue destruction without abscess formation. Although abscess formation and extensive tissue destruction are more likely to occur in IE caused by virulent bacteria, it may also occur in IE caused by oral streptococci especially when the diagnosis has been delayed.

\section{Antibiotic prophylaxis of IE}

The rationale for antibiotic prophylaxis to prevent the acquisition of IE depends on two premises - firstly, certain procedures, especially dental procedures, result in bacteraemia with organisms that commonly cause IE; and secondly, that certain cardiac conditions, both congenital and acquired, predispose to IE. Hence, the need to cover at risk procedures in at risk patients has been accepted clinical dogma for half a century. There are numerous national guidelines, including those from the UK devised by the BSAC working party; they differ mainly in detail not in principle. ${ }^{7}$ Overall, compliance with the guidelines is generally known to be poor for dental procedures, ranging from $15-35 \%$, and there are no data on compliance for the other procedures for which prophylaxis is recommended. The value of such prophylaxis in the prevention of IE has been questioned for some years as there is little or no objective evidence that it is effective in preventing IE; it is likely that spontaneous bacteraemias from chewing and various oral hygiene practices may be more significant in the pathogenesis of IE than certain dental procedures. Four studies of patients with IE during the last 16 years either fail to show a dental connection or can only show a small one, though the study designs are open to criticism. ${ }^{8}$ More recently a report from the USA suggested that the risk of IE after dental treatment is virtually nil. ${ }^{9}$

Some have argued that the danger of an anaphylactic reaction from penicillin/amoxicillin is much greater than the risk of IE, and it has been suggested that patients receiving penicillin/amoxicillin prophylaxis to prevent IE are five times more likely to die from an anaphylactic reaction to the drug than to die from contracting IE. There is also the potential for the selection of antibiotic resistance by the continued and repeated use of antibiotic prophylaxis. It has been estimated that a randomised placebo controlled study to assess the efficacy of antibiotic prophylaxis in dentistry would require at least 6000 at risk patients and would understandably encounter ethical objections, not least because of the ever present spectre of litigation. Perhaps, as David Durack has suggested, it is "time to scale back" and re-think the recommendations for dental prophylaxis though this would require a re-think of titanic proportions. It will be clear from the foregoing that there are cogent reasons for questioning dental prophylaxis; this applies even more so to non-dental prophylaxis 
such as that for many urological procedures. There is no evidence of efficacy and the antibiotics recommended for prophylaxis are parenteral and potentially toxic (for example, gentamicin and vancomycin in patients allergic to penicillin). It would thus seem reasonable to cease to recommend prophylaxis for these indications until there is a trial demonstrating efficacy, a formidable undertaking.

1. Durack DT, Lukes AS, Bright DK, and the Duke Endocarditis Service. New criteria for the diagnosis of

infective endocarditis: utilization of specific
echocardiographic findings. Am J Med 1994;96:200-9.

- The Duke criteria have been accepted internationally and no paper on IE would be acceptable without this assessment. The Duke criteria replaced the earlier case definitions introduced by von Reyn and colleagues in 1981.

2. Lamas CC, Eykyn SJ. Suggested modifications to the Duke criteria for the clinical diagnosis of native valve and prosthetic valve endocarditis: analysis of 118 pathologically proven cases. Clin Inf Dis 1997;25:713-9.

- Assessment of the Duke criteria in a large number of pathologically proven cases with suggestions for additional minor criteria that improved diagnostic sensitivity while retaining specificity.

3. Working Party of the British Society for Antimicrobial Chemotherapy. Antibiotic treatment of streptococcal, enterococcal and staphylococcal endocarditis. Heart

1998;79:207-10.

- The most recent UK guidelines on treatment. They cover treatment of the organisms that cause most cases of IE. Treatment of infections caused by unusual organisms cannot be expected from general guidelines. These guidelines, in contrast to the earlier ones (1985), no longer recommend either the $\mathrm{MBC}$ or serum bactericidal titrations.

4. Wilson WR, Thompson RL, Wilkowske CJ, et al. Short-term therapy for streptococcal infective endocarditis.
Combined intramuscular administration of penicillin and streptomycin. JAMA 1981;245:360-3.

- Although there are earlier studies of short course regimen this study found no relapses after two weeks of treatment in 91 patients. Its results seem to have been largely ignored in the UK.

5. Korzeniowski O, Sande MA and the National Collaborative Endocarditis Study Group. Combination antimicrobial therapy for Staphylococcus aureus endocarditis in patients addicted to parenteral drugs and in nonadddicts. A prospective study. Ann Intern Med 1982;97:496-503.

- One of the few studies to try and assess the efficacy of One of the few studies to try and assess the efficacy of
combination therapy in IE both in IVDU and non-IVDU and showing only a marginal benefit from the addition of gentamicin and more renal toxicity.

6. Douglas A, Moore-Gillon J, Eykyn S. Fever during treatment of infective endocarditis. Lancet 1986; i:1341-3.

- A study of 83 cases of culture positive NVE with persistent or recurrent fever during treatment that emphasises that the most common cause for this is extensive infection of the valve ring requiring surgery.

7. Working Party of the British Society for Antimicrobial Chemotherapy. Recommendations for endocarditis prophylaxis. J Antimicrob Chemother 1993;31:437-8. - These are the most recently published UK guidelines and feature in the British National Formulary and the Dental Formulary. They need updating and revising but are they are used in all litigation concerning prophylaxis.

8. Seymour RA, Lowry R, Whitworth JM, et al. Infective endocarditis, dentistry and antibiotic prophylaxis; time for a rethink? Br Dent J 2000;189:610-6.

- A useful and stimulating review of the current evidence that links dental treatment to IE and an appraisal of the risks of antibiotic prophylaxis.

9. Strom BL, Abrutyn E, Berlin JA, et al. Dental and cardiac risk factors for endocarditis. A population-based, case-control study. Ann Intern Med 1998;139:761-9.

- A major multicentre study that showed that dental treatment did not seem to be a risk factor for IE even in patients with valvar abnormalities whereas cardiac abnormalities are a strong risk factor. 